\section{0-456 EVALUATION OF EXPOSURE TO PESTICIDES THROUGH THE USE OF EFFECT BIOMARKERS IN RURAL WORKERS AND RURAL RESIDENTS IN RIO DE JANEIRO STATE - BRAZIL}

Marcia Sarpa de Campos Mello, 'Juliana Amazonas, Paula V Baptista, Katia Poça, Arthur Schilithz, Ubirani Otero, Sergio R Alves. ${ }^{1}$ FIOCRUZ, Brazil

\subsection{6/OEM-2021-EPI.100}

Introduction Brazil is one of the world's largest consumers of pesticides and this intense use impacts not only the environment but also exposes a wide range of individuals, such as rural workers who are occupationally exposed to pesticides and also the residents of the rural area environmentally exposed.

Objectives To evaluate occupational exposure to pesticides and to identify the neurotoxic and genotoxic effects in rural workers (RW) and rural residents (RR).

Methods A sectional epidemiological study was carried out with 104 RW and 23 RR of the city of Casimiro de Abreu (RJ/Brazil). A comparison group (CG) was formed with 103 residents of the urban area of the same city. Genotoxic analyses, through the comet assay and micronucleus test (MN), and the evaluation of the activity of cholinesterase enzymes (AChE and $\mathrm{BChE}$ ), were performed.

Results A reduction in cholinesterase enzyme activity was observed, mainly for butyrylcholinesterase of RW and RR when compared to CG (RW = 3856.40; RR = 3956.04; CG $=4359.57 ; \mathrm{p}=0.002)$. An increase in genotoxic effects in RW when comparing CG were observed (comet assay: RW = 21; $\mathrm{CG}=10 ; \mathrm{p}<0.001$ and $\mathrm{MN}$ number: $\mathrm{RW}=6.50 ; \mathrm{CG}$ $=3.00 ; \mathrm{p}<0.001)$, demonstrating that individuals occupationally exposed to pesticides are more likely to have genotoxic effects when compared to non-exposed individuals.

Conclusion The findings from this research will serve to support the execution of programs to monitor populations exposed to neurotoxic and genotoxic substances and allow the development of strategies for the prevention, control and surveillance of effects generated by occupational and environmental exposures to pesticides.

\section{Policy and Regulation}

\section{0-48 POLICY ISSUES ON WORK AND EMPLOYMENT IN SMALL SCALE MINING INDUSTRIES IN THE PHILIPPINES}

${ }^{1}$ Sophia Francesca Lu. 'University of the Philippines Diliman, Philippines

\subsection{6/OEM-2021-EPI.101}

Objective Small scale mining (SSM) has contributed 14\% of the total Gross Domestic Product (GDP) of the Philippines. The aim of the study is to look into policy issues on work and employment of small scale miners in the Philippines.

Methods The data were based on gray literature, peerreviewed journals, databases, government statistics, and secondary literature on major mining disasters in the Philippines, and the impact of regulation or lack thereof in this industry.

Results Research findings show that mining work is artisanal, with use of simple tools, and lack of sophisticated equipment and processes. There is massive use of toxic chemicals such as cyanide and mercury that have been associated with adverse health problems. The study review showed certain health issues due to cyanide and mercury poisoning from mining such as rapid breathing, gasping, tremors, convulsions, headache, dizziness and thyroid enlargement and eventually death, and children ages 17 years old and below experienced cough, wheezing, shortness of breath. In Western Mindanao, workers were found to be exposed to high levels of mercury. Gastrointestinal complaints of the workers were significantly associated with elevated hair methylmercury levels. Despite these, the government has no existing specific regulation and monitoring system for safety and health among small scale miners in the Philippines. Other policy issues included the following-that small scale mining is unregulated and unrecognized, absence of social safety net protection for the sector.

Conclusion Based on the research and policy reviews, there is a need to integrate the small scale mining into the labour economy so as to regulate and prevent hazardous work practices.

\section{0-70 EVOLUTION OF HEALTH INEQUALITIES IN THE CENTRAL AMERICAN WORKING POPULATION, 2011 - 2018}

${ }^{1}$ Michael Silva-Peñaherrera, George L Delclos, David Gimeno Ruiz de Porras, Marianela Rojas Garbanzo, Pamela Merino-Salazar, Maria Lopez-Ruiz, Fernando G Benavides. ' Center for research in Occupational Health, Spain

\subsection{6/OEM-2021-EPI.102}

Objective Monitoring of occupational health inequities is central to establishing effective public policies. The Central America Working Conditions Survey (ECCTS, by its Spanish acronym) was conducted in 2011 and 2018. This study analyzes changes, between these two periods, in the health inequality gap of workers in the six Spanish-speaking Central American countries, by gender, age, education level, occupation, and geographic area.

Methods The ECCTS is a cross-sectional survey of a nationally representative sample of workers (12,024 in 2011 and 9,030 in 2018), age $\geq 18$ years, in formal or informal employment. We calculated the prevalence of poor self-perceived health (SPH) and its 95\% confidence interval by country, age, education level, occupational category, and geographic area; differences between 2011 and 2018 were measured using chisquare statistics. We also measured absolute and relative population attributable risks, the Kuznets ratio and weighted Keppel index as inequality measures. All analyses were stratified by sex.

Results Overall, the health of the population in this region improved. Poor SPH decreased from $32 \%$ to $29 \%$ in women, and from $33 \%$ to $30 \%$ in men. The health inequalities gap among occupational groups remains wide. In women, the gap increased by occupation and geographic area, and decreased by education level. Among men, we found no statistically significant changes in the gap. Inequality between countries increased, evidenced by an increase in the Keppel index from $22 \%$ to $39 \%$ in women and from $20 \%$ to $29 \%$ in men.

Conclusion A general improvement in the health status was observed, but there was no progress in closing the health gap among occupational groups, and the gap between countries grew significantly. This study is the first to benchmark surveillance information that may contribute to developing, 